\title{
Adapting prior knowledge activation: Mobilisation, perspective taking, and learners' prior knowledge
}

Citation for published version (APA):

Wetzels, S. A. J., Kester, L., \& van Merrienboer, J. J. G. (2011). Adapting prior knowledge activation: Mobilisation, perspective taking, and learners' prior knowledge. Computers in Human Behavior, 27(1), 1621. https://doi.org/10.1016/j.chb.2010.05.004

Document status and date:

Published: 01/01/2011

DOI:

10.1016/j.chb.2010.05.004

Document Version:

Publisher's PDF, also known as Version of record

Document license:

Taverne

Please check the document version of this publication:

- A submitted manuscript is the version of the article upon submission and before peer-review. There can be important differences between the submitted version and the official published version of record.

People interested in the research are advised to contact the author for the final version of the publication, or visit the DOI to the publisher's website.

- The final author version and the galley proof are versions of the publication after peer review.

- The final published version features the final layout of the paper including the volume, issue and page numbers.

Link to publication

\footnotetext{
General rights rights.

- You may freely distribute the URL identifying the publication in the public portal. please follow below link for the End User Agreement:

www.umlib.nl/taverne-license

Take down policy

If you believe that this document breaches copyright please contact us at:

repository@maastrichtuniversity.nl

providing details and we will investigate your claim.
}

Copyright and moral rights for the publications made accessible in the public portal are retained by the authors and/or other copyright owners and it is a condition of accessing publications that users recognise and abide by the legal requirements associated with these

- Users may download and print one copy of any publication from the public portal for the purpose of private study or research.

- You may not further distribute the material or use it for any profit-making activity or commercial gain

If the publication is distributed under the terms of Article $25 \mathrm{fa}$ of the Dutch Copyright Act, indicated by the "Taverne" license above, 


\title{
Adapting prior knowledge activation: Mobilisation, perspective taking, and learners' prior knowledge
}

\author{
Sandra A.J. Wetzels ${ }^{\mathrm{a}, *}$, Liesbeth Kester $^{\mathrm{a}}$, Jeroen J.G. van Merriënboer ${ }^{\mathrm{a}, \mathrm{b}}$ \\ ${ }^{a}$ Centre for Learning Sciences and Technologies / Netherlands Laboratory for Lifelong Learning, Open Universiteit in the Netherlands, Heerlen, The Netherlands \\ ${ }^{\mathrm{b}}$ School of Health Professions Education, Maastricht University, Maastricht, The Netherlands
}

\section{A R T I C L E I N F O}

\section{Article history:}

Available online 31 May 2010

\section{Keywords:}

Prior knowledge activation

Mobilisation

Perspective taking

Prior knowledge

\begin{abstract}
A B S T R A C T
This study investigates the effects of two prior knowledge activation strategies, namely, mobilisation and perspective taking, on learning. It is hypothesised that the effectiveness of these strategies is influenced by learners' prior domain knowledge. More specifically, mobilisation is expected to be the most effective activation strategy at lower levels of prior knowledge. Mobilisation is a bottom-up oriented strategy that serves a broad stage-setting function. It provides learners with a relevant context in which new information can be integrated, which might be especially beneficial for learners with lower levels of prior knowledge to help them extend their limited knowledge base. As prior knowledge increases, perspective taking is expected to become the most effective strategy for activating learners' prior knowledge. Perspective taking is a top-down oriented strategy that results in the activation of a corresponding schema. This schema guides the selection and processing of information relevant to the schema, which might especially support learners with higher levels of prior knowledge to refine their already elaborated knowledge base. The effectiveness of the activation strategies (in terms of learning task performance) was indeed influenced by learners' prior knowledge in the hypothesised direction.
\end{abstract}

(c) 2010 Elsevier Ltd. All rights reserved.

\section{Introduction}

Prior knowledge activation has strong facilitative effects on learning. Chi, de Leeuw, Chiu, and LaVancher (1994), for example, activated students' prior knowledge by encouraging them to selfexplain each sentence of an expository text about the human circulatory system. Students in the control group read the same text twice without being asked to self-explain. Before and after reading the text, students' knowledge about the circulatory system was assessed. Students who relied on their prior knowledge while selfexplaining revealed higher knowledge gains than students who simply read the text twice. Prior knowledge activation in this case by means of self-explanation - facilitated the integration of information presented in the text with existing knowledge, which benefitted learning and understanding.

The present study investigates the effects of two prior knowledge activation strategies, namely, mobilisation and perspective taking. More specifically, it is investigated how the effectiveness of these activation strategies is influenced by learners' prior knowledge. The structure of the introduction is as follows. First,

\footnotetext{
* Corresponding author. Address: Open Universiteit in the Netherlands, Centre for Learning Sciences and Technologies/Netherlands Laboratory for Lifelong Learning, P.O. Box 2960, 6401 DL, Heerlen, The Netherlands. Tel.: +31 455762913.

E-mail address: sandra.wetzels@ou.nl (S.A.J. Wetzels).
}

the facilitative effects of prior knowledge activation on learning are described. Here, special attention is paid to the effects of mobilisation and perspective taking. Second, it is hypothesised how the effectiveness of mobilisation and perspective taking is influenced by learners' prior knowledge.

\section{Prior knowledge activation}

If learners activate their prior knowledge, this knowledge is brought from long-term memory to working memory providing learners with what Mayer (1979, p. 134) called “....an assimilative context of existing knowledge...". However, the availability of prior knowledge is not sufficient to reach higher learning outcomes. Learners should actively use the available prior knowledge by establishing relationships between the assimilative context held in working memory and new information (Mayer, 1979). So, prior knowledge provides learners with a relevant context in which new information can be integrated.

In line with Chi et al. (1994), many studies have provided evidence for a strong positive impact of prior knowledge activation on learning. De Grave, Schmidt, and Boshuizen (2001) used problem-based discussion to activate students' prior knowledge. Students collaboratively discussed a problem of blood pressure regulation or of vision, and tried to find explanations for these problems relying on their prior knowledge. Students who 
discussed the process of blood pressure regulation recalled more information from a text about this topic than the control group that discussed the text-irrelevant topic of vision. However, it cannot be excluded that these beneficial effects were also influenced by the group discussion process that might have provided some group members with new information. To prevent this possible confounding effect, in the present study, mobilisation and perspective taking are used in which learners activate their prior knowledge individually. These strategies are outlined in the following sections.

\subsection{Mobilisation}

A well-known technique for activating prior knowledge is mobilisation where learners are encouraged to bring to mind all knowledge they have in a certain domain (Peeck, 1982). Machiels-Bongaerts, Schmidt, and Boshuizen (1993) asked students in two experimental groups to mobilise either names of US states or names of US presidents. A control group mobilised names of composers. Subsequently, all students studied a list containing the names of 32 US states and presidents. Each item on the list was presented for a fixed amount of time. Both experimental groups showed higher recall scores than the control group. This higher recall was entirely caused by enhanced recall of items of the mobilised category; the presidents group recalled more president names than the group that mobilised state names or the control group, whereas the states group outperformed both other groups in recall of state names. These findings indicate that mobilising prior knowledge specifically facilitates the recall of information relevant to the activated knowledge.

In another study, Machiels-Bongaerts, Schmidt, and Boshuizen (1995) encouraged students to mobilise all knowledge they had about the fishery policy of the European Union and its consequences. A control group activated prior knowledge about a neutral topic (i.e., tennis). Subsequently, all students studied a text describing the effects of the restrictive EU fishery policy on a small imaginary fishermen's village. The text contained information about the background of the fishery policy and its consequences (e.g., a rise in unemployment) that were expected to correspond to the activated prior knowledge of the experimental group. Furthermore, the text contained information that provided additional, new information (e.g., an alternative income source) that became important in light of the activated prior knowledge. The experimental group outperformed the control group in recall of information from the text. This higher recall was caused by enhanced recall of information that was explicitly activated and of the new information that became relevant in the context of the activated knowledge. By establishing relations between the activated prior knowledge and the new information, the integration of the new information into the existing knowledge base is facilitated (Mayer, 1979). So, mobilisation enables learners to bridge the gap between their prior knowledge and new information provided to them with beneficial effects on learning.

\subsection{Perspective taking}

Another strategy used to activate learners' prior knowledge is perspective taking. This strategy is often investigated in the context of text processing and comprehension research where participants are assigned a perspective from which a text should be read. In an old study, Pichert and Anderson (1977), for example, found facilitative effects of assigning a perspective from which a text had to be read. The text described the adventures of two boys in one of the boys' homes while they were skipping school. It contained information that was relevant from the perspective of a po- tential homebuyer or a burglar. Before reading the passage, participants were instructed to take the perspective of either a homebuyer or a burglar, which was assumed to activate prior knowledge corresponding to the assigned perspective. After reading the text, a free recall test was administered. It was shown that information that was relevant to the assigned perspective was recalled better than perspective-irrelevant information.

These results were replicated by Goetz, Schallert, Reynolds, and Radin (1983) who used an expanded version of the skipping school passage. Again, participants read the story from the perspective of a homebuyer or a burglar. After studying the text, participants rated the importance of sentences and tried to recall everything they could remember from the text. Text elements relevant to participants' assigned perspective were rated more important and were recalled best. For the group that took the burglar perspective, burglar sentences were more important and recalled better than homebuyer sentences, whereas the opposite pattern was found for the group that read the text from the perspective of a prospective homebuyer. In addition, readers spent more time on sentences containing information that was relevant to their assigned perspective.

Assigning a perspective from which a text should be read results in the activation of an appropriate schema that guides subsequent information processing. As a result of activating a particular schema, a distinction is made between information that is relevant to the previously activated schema and information that is irrelevant in this respect. Selective attention is given to information corresponding with the previously activated knowledge. This results in the selection and in-depth processing of the schema-relevant information, which consequently leads to higher recall of that information. In contrast, information that is not in line with the assigned perspective will be ignored, and consequently not retrieved from memory (Pichert \& Anderson, 1977).

It should be noted that perspective taking is used in a slightly different context in the present study than in the traditional text research. The perspective does not have to be kept in mind when being confronted with the learning materials (e.g., while reading a text) but during prior knowledge activation before the learning materials are presented. However, in this context, the assigned perspective is also assumed to activate a corresponding schema that subsequently guides prior knowledge activation.

\section{Mobilisation, perspective taking, and learners' prior knowledge}

Although both mobilisation and perspective taking have beneficial effects on learning, the way these effects are brought about seem to differ. Mobilisation is a bottom-up oriented strategy; learners can freely activate a set of concepts that are only loosely connected and have not yet developed into a coherent knowledge structure. When relationships are established between the activated set of concepts (i.e., framework) and the newly provided information, the information can be integrated in the framework (Ginns, Chandler, \& Sweller, 2003; Kintsch, 1988). So, mobilisation serves a broad stage-setting function that provides learners with a relevant context (Peeck, van den Bosch, \& Kreupeling, 1982), which helps them to extend their prior knowledge (Kintsch, 1988). Perspective taking, in contrast, is a top-down oriented strategy. Prior knowledge activation by means of taking a specific perspective results in the activation of a corresponding schema. This schema already represents relevant concepts and their interrelations (Anderson, 1990). The activated schema guides the selection and processing of information relevant to this schema (Pichert \& Anderson, 1977), which is subsequently used to fill in gaps or update the schema. Therefore, perspective taking supports learners to 
further refine their prior knowledge represented in the activated schema.

Because of the difference in the information processing procedures (i.e., bottom-up, top-down) elicited by mobilisation and perspective taking, the effectiveness of these strategies is assumed to be influenced by learners' prior domain knowledge. More specifically, mobilisation seems especially suitable for learners with lower levels of prior knowledge, whereas perspective taking is assumed to be more suitable as learners' prior knowledge increases. At lower levels of prior knowledge, learners' prior knowledge consists of a set of loosely connected concepts (Kintsch, 1988). If learners establish relationships between this set of concepts and new information provided to them, their prior knowledge increases (Ginns et al., 2003). Therefore, learners with lower levels of prior knowledge are assumed to benefit most from mobilisation, a strategy that provides them with a relevant context and helps them to elaborate on and extend their limited knowledge base. With increasing prior knowledge, however, learners might benefit most from a strategy that enables them to further refine their already elaborated knowledge base (i.e., schema) by filling in gaps and updating the schema (Goetz et al., 1983). Therefore, learners with higher levels of prior knowledge might benefit most from a top-down oriented strategy such as perspective taking.

In sum, it is hypothesised that the effectiveness of mobilisation and perspective taking is influenced by learners' prior knowledge in the biology domain. More specifically, it is expected that mobilisation is most beneficial for learners at lower levels of prior knowledge. As prior knowledge increases, the beneficial effects of mobilisation are expected to fade away and reverse. At higher levels of prior knowledge, perspective taking is expected to be the most beneficial strategy for activating learners' prior knowledge.

\section{Method}

\subsection{Participants}

Sixty-three students (24 males and 39 females; mean age $=18.59$ years, $\mathrm{SD}=3.88$ ) participated in this study. To guarantee differences in prior knowledge in biology, students from preuniversity education (i.e., eleventh graders, $n=42$ ) and students from higher education (i.e., nursing and physiotherapy students, $n=21$ ) were asked to participate. They were paid $€ 20$ for their participation. A multiple regression with the factors prior knowledge activation strategy (mobilisation, perspective taking) and prior knowledge was used. Prior knowledge was included as a continuous variable to investigate the influence of learners' prior knowledge on the effectiveness of mobilisation and perspective taking. Participants were randomly assigned to either the mobilisation condition $(n=32)$ or the perspective taking condition $(n=31)$.

\subsection{Materials}

\subsubsection{Prior knowledge test}

A prior knowledge test with questions on the circulatory system was administered to assess differences in prior knowledge. This paper-and-pencil test contained 30 multiple-choice questions with four answer options that measured knowledge and understanding of the circulatory system. Questions were related to how blood flows through the body and the heart (e.g., when does blood flow from the atria to the ventricles?), how the heart functions (e.g., how does the electrical system of the heart work?), and related issues (e.g., what is the most common cause for heart failure?). One point was given for each correctly answered question on the prior knowledge test resulting in a maximum score of $30(M=16.51, \mathrm{SD}=5.01)$. Participants' scores ranged from 8 to 27 . Reliability of the prior knowledge test was $\alpha=.76$ (Cronbach's alpha).

\subsubsection{Prior knowledge activation picture}

Prior knowledge activation was initiated by means of a picture that illustrated the electrical system and the functioning of the heart. This picture is presented in Fig. 1.

\subsubsection{Learning tasks}

The learning phase consisted of seven learning tasks, designed according to the principles of the four-component instructional design model (Van Merriënboer \& Kirschner, 2007). The first learning task was a worked-out example that contained the main principles necessary to solve the remaining learning tasks. It described how blood flows through the heart and the body, how the electrical system of the heart works, and how this electrical activity is related to the functioning of the heart. After the worked-out example that contained high built-in learner support, four tasks with diminishing learner support were presented. Learner support was provided by using two completion problems and two reversal problems. In the first completion problem, three solution steps were already given and participants had to complete the remaining three solution steps to solve the problem. In the second completion problem, all solution steps were given in a random order and had to be rearranged by the participants to solve the problem. In the Appendix, these two types of completion problems are illustrated by applying them to the same problem. The two reversal problems provided the correct solution to the participants, who had then to use backward reasoning to explain why this solution fitted the problem posed. For example, a graph that illustrated the volume of blood in the ventricles of the heart was presented. In addition, three illustrations of the heart that represented a particular phase in the heart cycle (i.e., systolic phase of the atria, systolic phase of the ventricles, and diastolic phase of the heart) were provided. The correct answer concerning which of these three illustrations represented the blood volume in the graph was given and participants had to

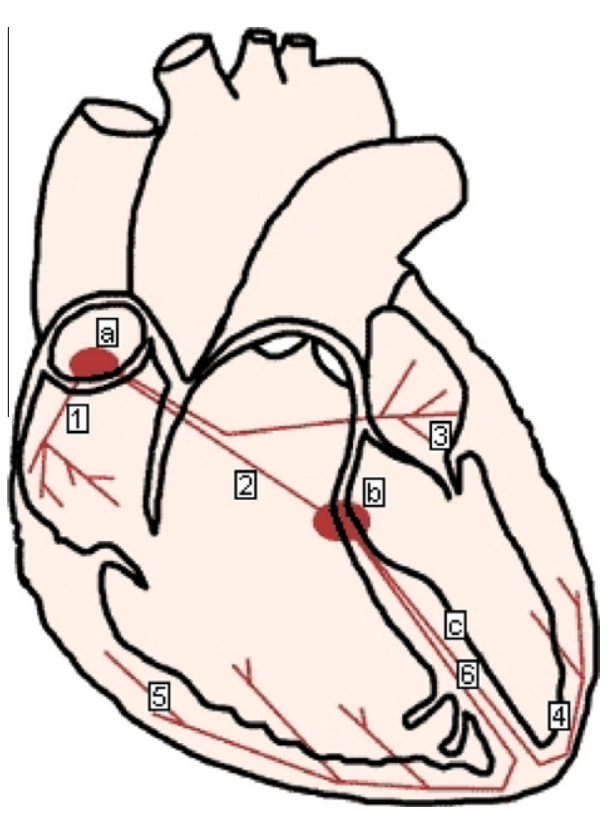

Fig. 1. Picture used to activate prior knowledge about the electrical system and the functioning of the heart. 
explain why this particular illustration fitted the graph. The last tasks in the sequence were two conventional problems without any support (i.e., no solution steps or correct solutions were provided). For example, participants were asked to describe the different activities that occur during the systolic phase of the ventricles.

\subsubsection{Transfer test tasks}

The test phase consisted of six transfer tasks. These transfer tasks required participants to apply the principles learned during the learning phase, and were used to assess participants' knowledge and understanding of the electrical system and the functioning of the heart. For example, participants had to explain how shrinking of the valves between the left atrium and ventricle as a result of endocarditis can lead to unconsciousness. Reliability of the transfer tasks was $\alpha=.59$ (Cronbach's alpha).

\subsection{Scoring}

\subsubsection{Performance}

For each learning task and transfer task, partial credits were given for a correct solution step. For example, if a task contained four solution steps, $1 / 4$ point was given for each correct solution step resulting in a maximum score of 1 for each task. The maximum scores in both the learning phase and the transfer phase were six.

\subsubsection{Mental effort}

Mental effort was measured using the mental effort rating scale of Paas (1992). Participants rated their invested mental effort on a 9-point scale ranging from 'very, very little effort' (1) to 'very, very much effort' (9). This measure provided an indication of how much mental effort participants had to invest to complete the prior knowledge test, activate their prior knowledge, and solve each learning task and transfer task.

\subsubsection{Time-on-task}

For each learning task and transfer task, time to solve the task was automatically recorded.

\subsubsection{Activated knowledge}

Activated knowledge was measured by analysing think-aloud protocols recorded in a randomly selected subset $(n=15)$ of all participants. The protocols were registered during prior knowledge activation using a headset and Audacity version 1.2.6 (http://audacity.sourceforge.net), and provided information on the knowledge that was activated by the participants. The think-aloud protocols were scored according to a coding scheme for determining relevancy and correctness of the activated prior knowledge. This coding scheme contained all important concepts necessary for solving learning tasks and transfer tasks. The worked-out example was used to distinguish these important concepts (e.g., Purkinje fibers, ventricles) and relations between these concepts (e.g., electrical activity in the His bundle is conducted to the Purkinje fibers, blood flows from the ventricles to the pulmonary artery and the aorta if the ventricles contract). Two independent raters scored the number of concepts, the number of relations, and the number of correct relations using the coding scheme. Interrater reliability was $r=.97$ (intraclass correlation).

\subsection{Procedure}

To avoid potential interference with prior knowledge activation, the prior knowledge test was administered at least 5 days before the experiment. In the experimental session, prior knowledge was activated once before participants started to work on the tasks. Prior knowledge activation was initiated by the prior knowledge activation picture that illustrated the electrical system and the functioning of the heart. This picture was presented on a computer screen for $5 \mathrm{~min}$. Instructions given to participants depended on the assigned condition. Participants in the mobilisation condition were instructed as follows: “. . .bring to mind everything you know about the electrical system and the functioning of the heart using the picture presented on the screen. How does the electrical system of the heart work? And what happens in the heart as a result of the electrical activity? Try to establish relations between the different things you already know..." Participants in the perspective taking condition were given the same instructions but were additionally encouraged to "...take the perspective of a blood cell that travels through the heart and meanwhile explains how the different parts of the heart work together..." So, the perspective taking instructions used in this study could be considered as a structured way of mobilising prior knowledge from an assigned perspective. Think-aloud protocols were recorded for a subset of all participants.

Subsequently, the learning phase started. First, all participants studied the worked-out example by reading the provided information carefully. Then, participants completed the two completion problems, worked on the two reversal problems, and solved the two conventional tasks. After finishing the learning phase, the transfer phase started in which participants solved the transfer tasks.

Participants rated the amount of mental effort they had to invest (a) after completing the prior knowledge test, (b) after activating their prior knowledge by means of mobilisation or perspective taking, (c) after each learning task, and (d) after each transfer task. All tasks and mental effort scales were presented on the computer screen. Participants were allowed to work on the tasks at their own pace.

\section{Results}

Multiple regression analyses were used to analyse the effects of mobilisation and perspective taking on performance and mental effort depending on learners' prior knowledge. The dichotomous variable prior knowledge activation strategy and the continuous variable prior knowledge were used in the analyses. In addition, the interaction term for prior knowledge and activation strategy was created by multiplying the centred scores on the prior knowledge test with the centred dichotomous values of the prior knowledge activation strategy (mobilisation $=-1$; perspective taking $=1$ ). The variables involved in the interaction were centred to prevent unstable estimations as a result of multicollinearity (Field, 2005). For all statistical tests, a significance level of .05 was maintained. Table 1 provides an overview of the means and standard deviations for performance, mental effort, and time-on-task for the learning phase and transfer phase.

Table 1

Means and standard deviations for performance, mental effort, and time-on-task.

\begin{tabular}{llllll}
\hline & \multicolumn{2}{l}{ Mobilisation } & & \multicolumn{2}{l}{ Perspective taking } \\
\cline { 2 - 3 } & $M$ & SD & & $M$ & SD \\
\hline Performance learning phase & 2.37 & 1.23 & & 2.80 & 1.43 \\
Performance transfer phase & 1.96 & 1.18 & & 2.37 & 1.19 \\
Mental effort learning phase & 5.46 & 1.22 & & 5.49 & 1.42 \\
Mental effort transfer phase & 5.88 & 1.33 & & 5.99 & 1.58 \\
Time-on-task learning phase (sec.) & 126 & 41 & & 146 & 47 \\
Time-on-task transfer phase (sec.) & 110 & 36 & & 133 & 44 \\
\hline
\end{tabular}




\subsection{Performance}

In order to investigate whether mobilisation and perspective taking had differential effects on performance depending on learners' prior knowledge, the interaction between prior knowledge and prior knowledge activation strategy was examined. An interaction effect between prior knowledge and activation strategy was found on learning task performance $(B=.057$, SE $B=.027, \beta=.213, p<.05)$. At lower levels of prior knowledge, activation through mobilisation was most beneficial for learning task performance. With increasing prior knowledge, this advantage of mobilisation faded away and reversed. At higher levels of prior knowledge, activation through taking a specific perspective became more beneficial than mobilisation (see Fig. 2).

In addition to the interaction effect of prior knowledge and activation strategy, a main effect of prior knowledge was found on learning task performance $(B=.161, S E B=.027, \beta=.600, p<.001)$ and transfer test performance $(B=.112$, SE $B=.025, \beta=.474$, $p<.001)$. As prior knowledge increased, performance on learning tasks and transfer tasks also increased.

\subsection{Mental effort}

When testing the influence of learners' prior knowledge on the effectiveness of mobilisation and perspective taking, no interaction between prior knowledge and activation strategy was found on mental effort. However, a main effect of prior knowledge was found on invested mental effort during the learning phase $(B=-.130$, SE $B=.029, \beta=-.496, p<.001)$ and the transfer phase $(B=-.122$, SE $B=.034, \beta=-.424, p<.01)$. As prior knowledge increased, mental effort invested while working on the learning tasks and transfer tasks decreased.

\subsection{Time-on-task}

In order to exclude possible confounding effects of differences in time spent working on tasks, the effects of time-on-task were also investigated. Main effects of prior knowledge $(B=2.790$, SE $B=.944, \beta=.335, p<.01)$ and prior knowledge activation strategy $(B=10.315$, SE $B=4.648, \beta=.249, p<.05)$ were found on time-on-task on the transfer tasks. More specifically, time-ontask spent working on transfer tasks increased with increasing

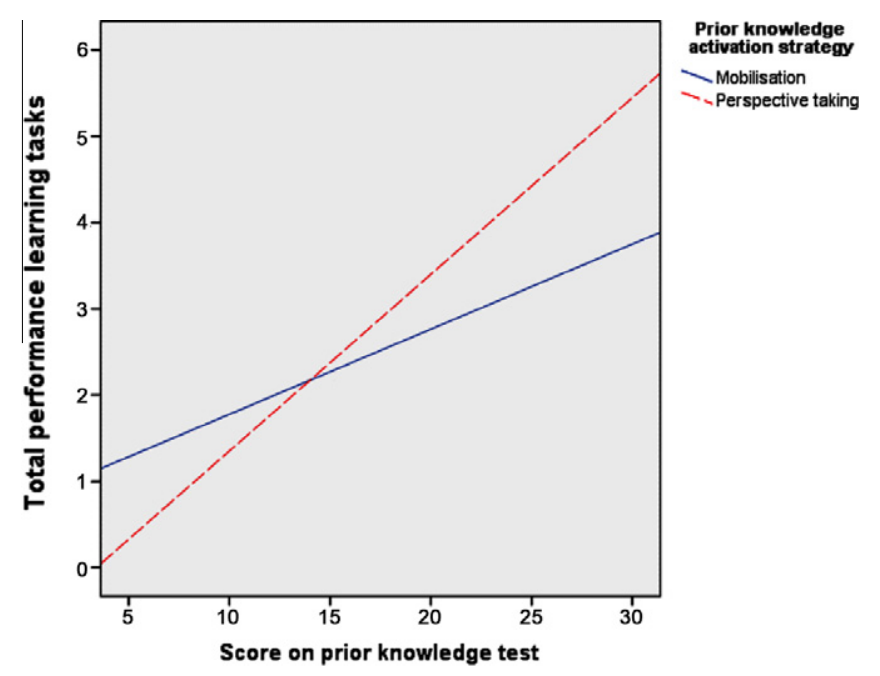

Fig. 2. Interaction effect between prior knowledge and prior knowledge activation strategy on learning task performance.
Table 2

Range, means, and standard deviations for the number of concepts, relations, and correct relations in the think-aloud protocols.

\begin{tabular}{llrl}
\hline & Range & M & SD \\
\hline Concepts & $0-23$ & 10.27 & 7.99 \\
Relations & $0-23$ & 6.55 & 8.13 \\
Correct relations & $0-23$ & 5.45 & 7.59 \\
\hline
\end{tabular}

prior knowledge. Furthermore, prior knowledge activation by means of perspective taking resulted in higher time-on-task spent working on transfer tasks than prior knowledge activation through mobilisation (see Table 1). The absence of a significant interaction effect on time-on-task implies that the interaction effect between prior knowledge and activation strategy on learning task performance was not influenced by differences in time-on-task.

\subsection{Activated knowledge}

The think-aloud protocols registered in a subset of all participants were investigated to gain more insights into the knowledge that was activated by participants. Table 2 provides the range, means, and standard deviations of the number of concepts, relations, and correct relations generated in the think-aloud protocols. A main effect of prior knowledge was found on the number of concepts $(B=.971$, SE $B=.247, \beta=.795, p<.01)$, relations $(B=1.050$, SE $B=.221, \beta=.845, p<.01)$, and correct relations $(B=.944$, SE $B=.225, \beta=.814, p<.01)$. As prior knowledge increased, participants activated more concepts, more relations, and more correct relations in the think-aloud protocols, that is, participants with higher levels of prior knowledge activated more and more correct prior knowledge than participants with lower levels of prior knowledge.

\section{Discussion}

The main goal of this study was to investigate the effects of two prior knowledge activation strategies (i.e., mobilisation and perspective taking) on learning depending on learners' prior knowledge. Results showed that the effectiveness in terms of performance of a specific activation strategy was influenced by the amount of prior domain knowledge learners already possessed. As expected, at lower levels of prior knowledge, learning task performance benefitted most if learners' prior knowledge was activated by means of mobilisation. With increasing prior knowledge, the effectiveness of mobilisation diminished and eventually reversed to perspective taking becoming the most effective activation strategy.

The results of this study seem to indicate that aligning the activation strategy to learners' prior knowledge supports the learning of new information but not the use and application of this information. Learning benefits if prior knowledge activation is tailored to learners' prior knowledge as was shown by a higher performance while working on the learning tasks. However, the use and application of the newly learned information did not seem to benefit from aligning prior knowledge activation to learners' prior knowledge since no beneficial effects were found in the transfer phase. The learning phase might have been too short to foster understanding of the principles learned, which would explain the absence of beneficial effects if learners had to apply the principles while working on the transfer tasks.

In addition to the finding that aligning prior knowledge activation to learners' prior knowledge benefits learning, results also showed that learners learn more effectively and efficiently as 
prior knowledge increases. More specifically, performance increased and mental effort decreased with increasing prior knowledge. In addition, learners generated more concepts and more (correct) relations in the think-aloud protocols as prior knowledge increased.

A limitation of this study is the relatively low number of participants. This might have had negative effects on the statistical power. Furthermore, although most item-total correlations were acceptable ( $r \geqslant .300$, see Field, 2005), the reliability of the transfer tasks was relatively low (i.e., $\alpha=.59$ ). This seems to indicate that not all transfer tasks assessed learners' knowledge and understanding of the electrical system and the functioning of the heart. Finally, it was difficult to determine whether participants were able to keep the perspective of the blood cell while activating their prior knowledge. In most think-aloud protocols, the blood cell was not explicitly mentioned. Therefore, it cannot be excluded that some participants did not follow the perspective taking instructions, which may imply that their activation process was similar to that of participants in the mobilisation condition. This should be more closely controlled for in future studies, for example, by asking participants afterwards how difficult it was to take and keep the assigned perspective in mind during prior knowledge activation.

A practical implication that follows from this study is that activating learners' prior knowledge is beneficial for learning because it provides learners with a framework in which new information provided by teachers or learning materials can be integrated. However, the activation of prior knowledge should be aligned to what learners already know about a certain topic. If learners are still novices in the domain, a bottom-up oriented strategy such as mobilisation should be used to provide learners with a relevant context to elaborate on their limited prior knowledge. With increasing prior knowledge, learners benefit most from a top-down oriented strategy such as perspective taking, which helps them to further refine their already elaborated knowledge base.

Future research may further explore the effects of mobilisation and perspective taking to provide more insights about the conditions in which these strategies are effective. In this study, the beneficial effects were confined to the learning phase. This implies that there is room for adapting and improving the use of these strategies in order to obtain long-term learning gains. Another interesting line of future research is related to the investigation of other prior knowledge activation strategies such as problem-based discussion or self-explanation. Is collaboratively discussing a problem about, for example, heart failure an effective strategy to activate learners' prior knowledge about the circulatory system? And assuming that the group discussing the problem consists of learners with different levels of prior knowledge, which members of the group (i.e., low, intermediate, or high prior knowledge learners) benefit most from this collaborative process of prior knowledge activation? Furthermore, because some learners might be provided with new information through problem-based discussion, it is important to investigate how genuine prior knowledge activation can be distinguished from learning from information provided by group members.

In sum, the present study provided evidence that the effectiveness of mobilisation and perspective taking is influenced by learners' prior domain knowledge. Aligning the activation strategy to what learners already know about a certain topic strengthens the beneficial effects of prior knowledge activation on learning.

\section{Appendix}

Types of completion problems.

The heart cycle consists of several phases and activities. Starting with the diastolic phase of the heart, which activities occur during one heart cycle?

Type I: Type II:

Complete solution Rearrange solution Correct solution steps steps

(1) blood flowing (1) closing of from atria to atrioventricular ventricles valves

(1) blood flowing from atria to

(2) electrical activity in sinus node

(3) contraction of atria

(2) blood flowing from atria to ventricles

(3) contraction of ventricles

(4) electrical activity in

(4) opening of atrioventricular valves
(5) node

(6)

(7)

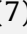

(5) electrical activity in atrioventricular node

(6) contraction of atria

(7) electrical activity in sinus node ventricles

(2) electrical activity in sinus node

(3) contraction of atria

(4) electrical activity in atrioventricular node

(5) contraction of ventricles

(6) closing of atrioventricular valves

(7) opening of arterial valves

\section{References}

Anderson, J. R. (1990). Cognitive psychology and its implications. New York: Freeman. Chi, M. T. H., de Leeuw, N., Chiu, M., \& LaVancher, C. (1994). Eliciting selfexplanations improves understanding. Cognitive Science, 18, 439-477.

De Grave, W. S., Schmidt, H. G., \& Boshuizen, H. P. A. (2001). Effects of problembased discussion on studying a subsequent text: A randomized trial among first year medical students. Instructional Science, 29, 33-44.

Field, A. (2005). Discovering statistics using SPSS (2nd ed.). London: SAGE Publications Ltd..

Ginns, P., Chandler, P., \& Sweller, J. (2003). When imagining information is effective. Contemporary Educational Psychology, 28, 229-251.

Goetz, E. T., Schallert, D. L., Reynolds, R. E., \& Radin, D. I. (1983). Reading in perspective: What real cops and pretend burglars look for in a story. Journal of Educational Psychology, 75, 500-510.

Kintsch, W. (1988). The role of knowledge in discourse comprehension: A construction-integration model. Psychological Review, 95, 163-182.

Machiels-Bongaerts, M., Schmidt, H. G., \& Boshuizen, H. P. A. (1993). Effects of mobilizing prior knowledge on information processing: Studies of free recall and allocation of study time. British Journal of Psychology, 84, 481-498.

Machiels-Bongaerts, M., Schmidt, H. G., \& Boshuizen, H. P. A. (1995). The effect of prior knowledge activation on text recall: An investigation of two conflicting hypotheses. British Journal of Educational Psychology, 65, 409-423.

Mayer, R. E. (1979). Twenty years of research on advance organizers: Assimilation theory is still the best predictor of results. Instructional Science, 8, 133-167.

Paas, F. (1992). Training strategies for attaining transfer of problem-solving skills in statistics: A cognitive-load approach. Journal of Educational Psychology, 84, 429-434.

Peeck, J. (1982). Effects of mobilization of prior knowledge on free recall. Journal of Experimental Psychology: Learning, Memory, and Cognition, 8, 608-612.

Peeck, J., van den Bosch, A. B., \& Kreupeling, W. J. (1982). Effect of mobilizing prior knowledge on learning from text. Journal of Educational Psychology, 74, 771-777.

Pichert, J. W., \& Anderson, R. C. (1977). Taking different perspectives on a story. Journal of Educational Psychology, 69, 309-315.

Van Merriënboer, J. J. G., \& Kirschner, P. A. (2007). Ten steps to complex learning. Mahwah, NJ: Lawrence Erlbaum Associates. 